\title{
La natación, el ciclismo y los deportes de raqueta se asociaron con disminución de la mortalidad global
}

Swimming, cycling and racquet sports were associated with decreased in all-cause mortality

\section{Objetivos}

Evaluar la asociación entre seis tipos diferentes de ejercicios/ deportes y la mortalidad por todas las causas y de causa cardiovascular.

\section{Diseño, lugar y pacientes}

Estudio de cohorte realizado en Escocia e Inglaterra que incluyó 80.306 individuos (54\% mujeres; media de edad $52 \pm 14$ años, de 30 a 98 años de edad) de la Encuesta de Salud de Inglaterra (HSE) y de Escocia (SHeS) de base poblacional que reclutan muestras anuales desde 1991 y 1995 respectivamente.

\section{Evaluación de factores pronósticos}

Se realizaron entrevistas en los hogares incluidos en la muestra, completando un cuestionario para evaluar actividad física como caminar, practicar deportes, y también acerca de actividades domésticas en las cuatro semanas previas a la entrevista. Para los deportes, que incluyó ciclismo (con cualquier propósito), na-
Oja P, y col. Br J Sports Med 2016;0:1-7. tación, ejercicios de aerobics, gimnasia/danza, running, fútbol/ rugby y deportes de raqueta (tenis, badmington y squash). Se pidió a los participantes que especificaran la frecuencia, duración e intensidad de la actividad.

\section{Medición de resultados principales}

Se registró mortalidad censando a los participantes supervivientes en Diciembre de 2009 (SHeS) o Febrero de 2011 (HSE) y las principales causas de muerte según la Clasificación Internacional de Enfermedades (CIE-10).

\section{Resultados Principales}

Se observaron reducciones significativas en la mortalidad por todas las causas en: ciclismo, natación, deportes de raqueta y ejercicios aeróbicos. Se encontraron asociaciones estadísticamente significativas para la participación en todas las actividades excepto para fútbol y running (Ver Tabla 1).

Tabla 1. Asociación entre ejercicios/deportes y la mortalidad por todas las causas y cardiovascular.

\begin{tabular}{|c|c|c|c|c|}
\hline \multirow[b]{2}{*}{ Deporte/ejercicio } & \multicolumn{2}{|c|}{$\begin{array}{l}\text { Mortalidad por todas las causas } \\
n=80.306\end{array}$} & \multicolumn{2}{|c|}{$\begin{array}{c}\text { Mortalidad cardiovascular* } \\
n=75.014\end{array}$} \\
\hline & HR & IC 95\% & HR & IC 95\% \\
\hline Ciclismo & 0,85 & 0,76 a 0,95 & 0,93 & 0,76 a 1,16 \\
\hline Natación & 0,72 & 0,65 a 0,80 & 0,59 & 0,46 a 0,75 \\
\hline Deportes de raqueta & 0,53 & 0,40 a 0,69 & 0,44 & 0,24 a 0,83 \\
\hline Aeróbicos & 0,73 & 0,63 a 0,85 & 0,64 & 0,45 a 0,92 \\
\hline Fútbol & 0,82 & 0,61 a 1,11 & 0,90 & 0,49 a 1,64 \\
\hline Running & 0,87 & 0,68 a 1,11 & 0,81 & 0,47 a 1,39 \\
\hline
\end{tabular}

*Se excluyeron individuos con ECV al inicio

\section{Conclusiones}

La participación en deportes específicos puede tener beneficios para la salud pública.

\section{Comentario}

Varios estudios han demostrado el efecto beneficioso de la actividad física para la salud. Una revisión sistemática, que incluyó 80 estudios con 1.338 .143 participantes, encontró el mayor beneficio en mortalidad por todas las causas para la práctica "vigorosa" de ejercicios y deportes $(\mathrm{RR}=0,78)^{1}$.

El presente estudio tiene como fortalezas el incluir una cohorte numerosa y medir resultados duros como la mortalidad. Los autores observaron reducciones clínica y estadísticamente significativas en la mortalidad por todas las causas para la participación en: ciclismo, natación, deportes de raqueta y aeróbicos. No se encontraron asociaciones significativas para la participación en fútbol y running. Si bien se realizaron ajustes por enfermedades crónicas, consumo de alcohol, problemas psicológicos, índice de masa corporal, tabaquismo, nivel educativo, enfermedad cardiovascular previamente conocida y otros tipos de actividad física; vale la pena mencionar cierta debilidad de este estudio relacionada con la evaluación limitada de la exposición, ya que sólo se evaluaba el recuerdo de actividad en las últimas cuatro semanas, sin tener en cuenta la variabilidad en el desarrollo del deporte, otras actividades previas que haya hecho el individuo en su vida, o el tiempo total de exposición, lo cual podría conducir a errores de clasificación de los participantes. En este sentido, encuentro más válido el resultado reportado en mortalidad que consideró la exposición englobando a todos los participantes que hacían ejercicios/deportes vs los que no hacían deporte: HR=0,73 (IC $95 \% 0,69$ a 0,$77 ; p<0,01)$.

\section{Conclusiones del comentador}

Considero que en nuestra práctica clínica cotidiana, independientemente de que una actividad pueda demostrarse como más beneficiosa que otra, debemos incentivar el desarrollo de actividad física, priorizando aquellas actividades o deportes que sean aceptables para la persona y que por sobre todo le generen placer.

Luciani L. La natación, el ciclismo y los deportes de raqueta se asociaron con disminución de la mortalidad global. Evid Act Pract Ambul. 2017;20(1):44. Comentado de: Oja P, y col. Associations of specific types of sports and exercise with all-cause and cardiovascular desease mortality: a cohort study of $\mathbf{8 0 . 3 0 6}$ British adults. Br J Sports Med, 2016;0:1-7. PMID 27895075. 